\title{
Charmonium spectrum with all-to-all propagators
}

\author{
K.J. Juge, A. Ó Cais, M.B. Oktay*, M.J. Peardon, S.M. Ryan \\ Trinity College Dublin \\ E-mail: ektayemaths.tcd.ie
}

\begin{abstract}
We present a first study of the charmonium spectrum on $N_{f}=2$ dynamical, anisotropic lattices. We take advantage of all-to-all propagators to build spatially extended interpolating operators to increase the overlap with states not easily accessible with point propagators, such as radially excited states of $\eta_{c}, \psi$ and $\chi_{c}$; D-waves and hybrid states. The full results are presented in the contribution by K.J. Juge, PoS(LAT2005)029.
\end{abstract}

XXIIIrd International Symposium on Lattice Field Theory

25-30 July 2005

Trinity College, Dublin, Ireland

${ }^{*}$ Speaker. 